\title{
DNA replication licensing control and rereplication prevention
}

\author{
Chonghua $\mathrm{Li}^{1}$, Jianping $\mathrm{Jin}^{1,2} \bowtie$ \\ ${ }^{1}$ Department of Biochemistry and Molecular Biology, The University of Texas Medical School at Houston, Houston, TX 77030, \\ USA \\ 2 The Graduate School of Biomedical Sciences, The University of Texas Health Science Center at Houston, Houston, TX 77030, \\ USA \\ $凶$ Correspondence: jianping.jin@uth.tmc.edu
}

Received January 6, 2010 Accepted January 18, 2010

\begin{abstract}
Eukaryotic DNA replication is tightly restricted to only once per cell cycle in order to maintain genome stability. Cells use multiple mechanisms to control the assembly of the prereplication complex (pre-RC), a process known as replication licensing. This review focuses on the regulation of replication licensing by posttranslational modifications of the licensing factors, including phosphorylation, ubiquitylation and acetylation. These modifications are critical in establishing the pre-RC complexes as well as preventing rereplication in each cell cycle. The relationship between rereplication and diseases, including cancer and virus infection, is discussed as well.
\end{abstract}

KEYWORDS DNA replication licensing, rereplication, protein modification

\section{INTRODUCTION}

In eukaryotic cells, the size of the genome ranges from $\sim 10^{7}$ up to $>10^{11} \mathrm{bp}$ (Lewin, 2000). Regardless of the size of the genome, all of the chromosomal DNA sequence must be precisely replicated once and only once in each cell cycle. Any genome over- or under-replication within a cell cycle will cause genome instability, which is the hallmark of cancer. To maintain genome integrity, cells have developed multiple mechanisms to assure a single duplication of the entire genome during each round of cell cycle.

DNA replication starts at multiple sites on each chromosome, called origins. In budding and fission yeasts, the sixsubunit origin recognition complex (ORC) binds to chromatin throughout the cell cycle. In higher eukaryotes, however,
$\mathrm{ORC}$ is released from chromatin in mitosis due to phosphorylation (for review, see DePamphilis, 2005; Arias and Walter, 2007). Upon exit from mitosis, ORC recognizes and binds to the origins. It then recruits a protein called cell division cycle 6 (Cdc6) followed by the association of another DNA replication factor called Cdt1. These proteins form a platform that facilitates loading of the mini chromosome maintenance (MCM) 2-7 DNA helicase complex onto chromatin. Together, ORC, Cdc6, Cdt1 and MCM2-7 constitute the prereplication complex (pre-RC) (for review, see Bell and Dutta, 2002). The process of pre-RC assembly on origins is also known as replication licensing, which is the key step in initiating DNA replication (Fig. 1). Inactivation of these licensing factors is critical for preventing reinitiation of DNA replication from the origins in the rest of the cell cycle.

Although different organisms have evolved various mechanisms to prevent rereplication, essentially all mechanisms target pre-RC components, mainly Cdc6 and Cdt1 (for reviews, see DePamphilis et al., 2006; Arias and Walter, 2007; Hook et al., 2007). In budding yeast, several independent pathways work redundantly to prevent relicensing in S, G2 and $M$ phases. These pathways include downregulation of the level of $\mathrm{Cdc} 6$ by ubiquitin-mediated proteolysis and by inhibition of Cdc6 transcription; inactivation of Cdc6 and ORC proteins by binding of specific inhibitors; and nuclear export of Cdt1 and MCM complex (reviewed by Arias and Walter, 2007). Phosphorylation of the pre-RC components by the cyclin dependent kinase (CDK) plays an essential role in regulating the pre-RC formation in budding yeast, which will be discussed later in this review.

In metazoans, Cdt1 is one of the major targets for prevention of rereplication. Unlike budding yeast, whose level of Cdt1 remains constant throughout the cell cycle, metazoan Cdt1 is negatively regulated by two ubiquitylation- 


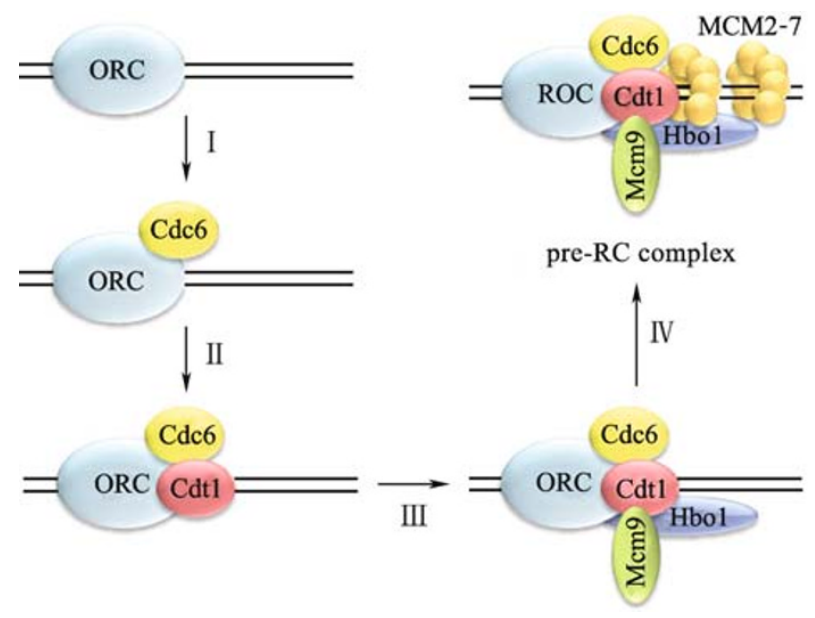

Figure 1 . The assembly of pre-RC complex during replication licensing in vertebrates. (I) In early $\mathrm{G} 1$ phase, Cdc6 is recruited to the origin through interaction with the ORC complex. (II) Subsequently, Cdc6 recruits Cdt1 to the origin. (III) Two positive regulators, $\mathrm{Mcm} 9$ and $\mathrm{Hbo} 1$, join the origin via interactions with $\mathrm{Cdt} 1$. It is not known whether $\mathrm{Mcm} 9$ and $\mathrm{Hbo} 1$ interact with each other. (IV) MCM2-7 complex is loaded onto the origin in late $\mathrm{G} 1$ phase. The prereplication complex (pre$\mathrm{RC}$ ) is assembled completely and the origin is ready to be fired in S phase.

mediated proteolysis pathways when cells progress into $\mathrm{S}$ phase (Fig. 2) (Li et al., 2003; Zhong et al., 2003; Arias and Walter, 2005; Nishitani et al., 2006; Kim and Kipreos, 2007). From late $\mathrm{G} 1$ to late $\mathrm{M}$ phase, Cdt1 is inactivated through interaction with a small protein, called geminin (Wohlschlegel et al., 2000; Tada et al., 2001). The association of Cdt1 with geminin blocks the recruitment of $\mathrm{MCM} 2-7$, which in turn prevents pre-RC assembly, thereby prohibiting DNA replication licensing (Fig. 2) (for review, see Arias and Walter, 2007). The binding of geminin also protects Cdt1 from ubiquitylation and proteolysis, ensuring that an adequate amount of Cdt1 is ready for replication licensing in the next round of cell cycle (Ballabeni et al., 2004). Although Cdt1 is the primary target for regulating replication licensing in metazoans, there is evidence suggesting that both ORC and Cdc6 are also regulated by phosphorylation and ubiquitylation to prevent relicensing (Fig. 2). Recently, a new MCM2-7 family member, MCM9, was found to be essential for MCM2-7 loading in vertebrates. MCM9 directly interacts with and positively regulates Cdt1 (Lutzmann and Mechali, 2008). Hbo1 (histone acetyltransferase bound to Orc1) has been also shown to be crucial for MCM2-7 recruitment (Miotto and Struhl, 2008). The requirement of $\mathrm{Mcm} 9$ and $\mathrm{Hbo} 1$ in pre-RC assembly adds an additional control on replication licensing in vertebrates (Fig. 1).

Among the various regulatory pathways described above, posttranslational modifications of the licensing proteins and their regulators have been shown to play indispensible roles

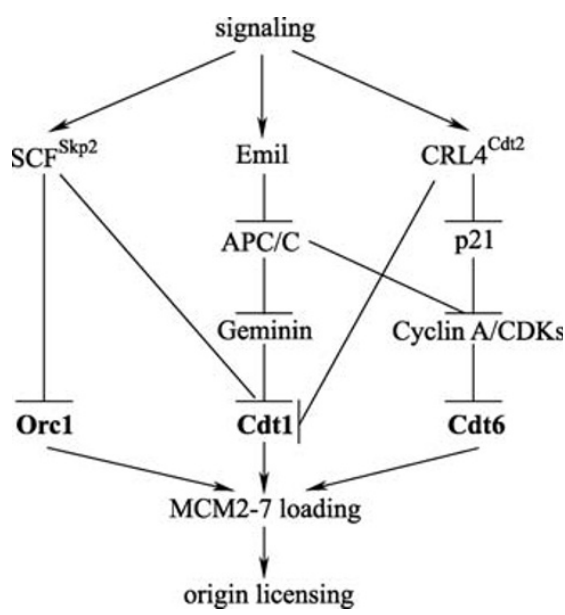

Figure 2. DNA replication licensing control in metazoan. The center of the DNA replication licensing control is the regulation of those DNA replication licensing factors, such as Cdt1, Cdc6 and Orc1, The licensing activity of Cdt1 is inhibited through direct interaction with Geminin and via ubiquitylation by two ubiquitin ligases, CRL4 ${ }^{\mathrm{Cdt} 2}$ and $\mathrm{SCF}^{\mathrm{Skp} 2}$ that also ubiquitylates Orc1 and directs its degradation. Cdc6 is phosphorylated by Cyclin A/CDKs (mainly Cdk2) and exported to cytoplasm, where it loses its licensing function. Cyclin A and Geminin are degraded via ubiquitylation by ubiquitin ligase, $\mathrm{APC} / \mathrm{C}$, which is inactivated by Emi1 in S and $\mathrm{G} 2$ phases. Cyclin/Cdk2 kinase activity is also suppressed by p21 that is controlled by CRL4 ${ }^{\text {Cdt2 }}$ via ubiquitylation and proteolysis. Dysregulation of those licensing factors can lead to rereplication and aneuploidy.

in preventing rereplication within a single cell cycle. The first part of this review summarizes the roles of protein phosphorylation, ubiquitylation and acetylation in regulating replication licensing. In the second part, we discuss how dysregulation of replication licensing is involved in diseases, such as cancer and virus infection, and the possibilities of aiming at the replication licensing system as therapeutic targets.

\section{PHOSPHORYLATION REGULATES DNA REPLICATION LICENSING}

Protein phosphorylation is a fundamental mechanism to assure proper progression of the cell cycle, including replication licensing. In fact, CDK activities play an essential role in controlling replication licensing in budding yeast, and almost every component of the pre-RC complex is subjected to phosphorylation regulation. For example, Orc2 and Orc6 are both phosphorylated by Cdc28/Cyclin B throughout the cell cycle, except in G1 phase (Nguyen et al., 2001). Cdc6 can be downregulated at the protein level by ubiquitylationand proteasome-mediated destruction when cells enter $\mathrm{S}$ phase (Drury et al., 1997), which requires Cdc28-dependent phosphorylation of Cdc6 (Elsasser et al., 1999). Cdc6 can 
also be inactivated by forming a stable complex with the CDK, $\mathrm{Cdc} 28 / \mathrm{Clb2}$, in $\mathrm{G} 2$ and $\mathrm{M}$ phases, and the interaction between Cdc6 and CDK depends on phosphorylation of the $\mathrm{N}$-terminal domain of Cdc6 (Mimura et al., 2004). Moreover, the MCM2-7 complex can be phosphorylated by $\mathrm{Cdc} 28$ and exported from the nucleus to the cytoplasm during S, G2 and M phases (Labib et al., 1999; Nguyen et al., 2000; Liku et al., 2005).

In metazoans, however, the regulation of ORC proteins and Cdc6 may be cell-type dependent. In Chinese hamster ovary cells $(\mathrm{CHO})$, Orc1 can be phosphorylated by Cyclin A/Cdk1 during $\mathrm{G} 2 / \mathrm{M}$ phase, which reduces its affinity for chromatin ( $\mathrm{Li}$ et al., 2004); whereas in HeLa cells, phosphorylation of Orc1 by Cyclin $\mathrm{A} / \mathrm{Cdk} 2$ directs Orc1 for $\mathrm{SCF}^{\mathrm{Skp} 2}$-dependent proteolysis in S phase (Mendez et al., 2002). Additionally, phosphorylation of Orc1 has been shown to direct the translocation of Orc1 into the cytoplasm and prevents pre$\mathrm{RC}$ formation in both $\mathrm{CHO}$ and HeLa cells (Saha et al., 2006). In several types of human cells, a portion of Cdc6 is phosphorylated by Cyclin A/Cdk2 and the phosphorylation is required for nuclear export of Cdc6 in S phase to prevent rereplication (Fig. 2) (Jiang et al., 1999; Petersen et al., 1999; Delmolino et al., 2001). A similar phenomenon was also observed in C. elegans (Kim et al., 2007). In human cells, phosphorylation of Cdt1 by CDKs is required for the ubiquitylation-dependent degradation pathway that is controlled by the $\mathrm{SCF}^{\mathrm{Skp} 2}$ ubiquitin ligase (Fig. 2) ( Liu et al., 2004; Sugimoto et al., 2004).

The role of phosphorylation in replication licensing control has also been extensively reviewed elsewhere (Bell and Dutta, 2002; Nishitani and Lygerou, 2002; DePamphilis et al., 2006; Arias and Walter, 2007; Kim and Kipreos, 2008). In general, phosphorylation of licensing proteins exerts its regulatory function by targeting the proteins for ubiquitylation and subsequent proteolysis, by mediating interaction with specific inhibitors, or by directing translocation of the proteins to cytoplasm to prevent pre-RC formation.

\section{UBIQUITYLATION CONTROLS DNA REPLICATION LICENSING}

One efficient way to prevent DNA rereplication is to downregulate the pre-RC components via proteolysis. Most proteolysis in the cell occurs via the 26S proteasome which requires prior ubiquitylation of the target proteins. Ubiquitylation involves covalent attachment of ubiquitin to the target proteins via a cascade of three enzymes, including an activating enzyme E1, a conjugating enzyme E2 and a ubiquitin ligase E3 (Pickart, 2004). Among the various E3 ligases, the Cullin-RING E3 superfamily (CRL) and the anaphase-promoting complex/cyclosome (APC/C) have been shown to play pivotal roles in DNA replication licensing control (Fig. 2).

In human cells, cell cycle-regulated Cdt1 proteolysis is controlled by two different ubiquitylation pathways. The first pathway is mediated by the $\mathrm{SCF}^{\mathrm{Skp} 2}$ ubiquitin ligase, a Cullin1-RING E3 ligase (Li et al., 2003; Nishitani et al., 2006). $\mathrm{SCF}^{\mathrm{Skp} 2}$ was shown to associate with and ubiquitylate Cdt1 in vivo. Overexpression of the substrate receptor Skp2 results in a decrease of Cdt1 protein levels, while knocking down of Skp2 by siRNA extends the half life of Cdt1 (Li et al., 2003). As mentioned above, the $\mathrm{SCF}^{\mathrm{Skp} 2}$-dependent ubiquitylation of Cdt1 occurs during $S$ and $G 2$ phases, and it depends on phosphorylation of Cdt1 by CDKs (Liu et al., 2004; Sugimoto et al., 2004; Takeda et al., 2005). Interestingly, however, Cdt1 ubiquitylation via $\mathrm{SCF}^{\mathrm{Skp} 2}$ has only been observed in human cells thus far. Alternatively, Cdt1 can be destroyed by a Cullin4 and Cdt2 (CRL4 $\left.{ }^{\mathrm{Cdt} 2}\right)$-dependent pathway that is conserved from yeast to human (Hu et al., 2004; Jin et al., 2006; Lovejoy et al., 2006; Nishitani et al., 2006; Ralph et al., 2006; Sansam et al., 2006). Unlike the SCF ${ }^{\text {Skp2 }}$-dependent pathway, the proteolysis of Cdt1 facilitated by CRL4 ${ }^{\mathrm{Cdt} 2} \mathrm{E} 3$ ligase is restricted to $S$ phase and requires chromatin-bound proliferating cell nuclear antigen (PCNA ${ }^{\text {Chromatin }}$ ) (Arias and Walter, 2006; Hu and Xiong, 2006; Senga et al., 2006), indicating that Cdt1 destruction is coupled with DNA replication. The CRL4 ${ }^{\text {Cdt2 }}$-dependent proteolysis of Cdt1 is also activated when DNA damage occurs (Jin et al., 2006). The two ubiquitylation pathways act independently to regulate the level of Cdt1 through the cell cycle and both contribute to DNA rereplication prevention.

The CDK inhibitor, p21, is also involved in regulating DNA replication licensing (Fig. 2) (Kim et al., 2008). As a CDK inhibitor, p21 protein plays multiple roles in many cellular processes. The best characterized function of $\mathrm{p} 21$ is to bind to and inhibit the activity of Cyclin/Cdk2 or Cyclin/Cdk4 complexes, which causes cell cycle arrest upon stress stimuli. It also interacts with PCNA and negatively regulates DNA replication and DNA repair (for review, see Prives and Gottifredi, 2008). The level of $p 21$ can be regulated at the transcriptional level as well as at the posttranscriptional level. It is well-known that p21 protein is subjected to proteasomeand ubiquitin-mediated degradation via the actions of the E3 ligases, including $\mathrm{SCF}^{\mathrm{Skp2}}$, APC/C, MDMX (Yu et al., 1998; Bornstein et al., 2003; Amador et al., 2007) and ubiquitinindependent, but proteasome-dependent pathway (Chen et al., 2007; Li et al., 2007). Recently, three groups simultaneously found that $\mathrm{p} 21$ is also degraded via ubquitylation by CRL4 ${ }^{\text {Cdt2 }}$ in human cells upon DNA damage treatment (Abbas et al., 2008; Kim et al., 2008; Nishitani et al., 2008). This p21 degradation pathway is independent of the one mediated by $\mathrm{SCF}^{\mathrm{Skp2}}$ (Abbas et al., 2008; Kim et al., 2008; Nishitani et al., 2008), and ubiquitylation depends on the interaction between p21 and PCNA ${ }^{\text {Chromatin }}$, which is similar to the CRL4 ${ }^{\mathrm{Cdt} 2}$-targeted degradation of Cdt1 (Abbas et al., 2008; Kim et al., 2008; Nishitani et al., 2008). Kim and colleagues (2008) also showed that the DNA rereplication phenotype induced in Cdt2 knockdown cells can be abolished 
by co-silencing $\mathrm{p} 21$. This suggests that $\mathrm{p} 21$ contributes to the rereplication that occurs when Cdt2 is inactivated. A similar result was observed in $C$. elegans, in which Cul4-Ddb1 ubiquitylation pathway ubiquitylates $\mathrm{CDK}$ inhibitor, $\mathrm{CKI}-1$, for degradation. Depletion of CKI-1 by RNAi can partially rescues the rereplication phenotype in a cul4 mutant (Kim and Kipreos, 2007). So, how does p21 regulate replication licensing? One possible downstream component of p21 in the licensing pathway is Cdc6. Previous studies indicated that Cdc6 is phosphorylated by CDK and can be exported to the cytoplasm in S and G2 phases in a phosphorylationdependent pathway (Jiang et al., 1999; Petersen et al., 1999; Delmolino et al., 2001). Since stabilization of p21 by inactivation of $\mathrm{Cdt} 2$ causes a modest defect in nuclear export of Cdc6, p21 may contribute to replication licensing by inhibiting CDK activities that regulate Cdc6 localization by phosphorylation (Kim et al., 2008). Alternatively, considering that CDKs have many other cellular targets, p21 may also regulate replication licensing through other $\mathrm{Cdc6}$-independent pathway.

As mentioned above, both Cdt1 and p21 degradation requires direct interaction with PCNA ${ }^{\text {Chromatin }}$. This interaction is mediated through a consensus PCNA-binding motif, called the PIP box (Moldovan et al., 2007; Havens and Walter, 2009). However, the PIP box alone is not sufficient to recruit CRL4 ${ }^{\text {Cdt2 }}$ to PCNA ${ }^{\text {Chromatin }}$, it also requires a positively charged amino acid at the +4 position in the PIP box which is conserved in human p21, Cdt1 and worm CKI-1 but not in other stable PIP box proteins (Havens and Walter, 2009). It was hypothesized that binding of the substrate with PCNA ${ }^{\text {Chromatin }}$ creates a specific surface that allows recruitment of CRL4 ${ }^{\text {Cdt2 }}$ ubiquitin ligase (Arias and Walter, 2006; Havens and Walter, 2009). The requirement of chromatinbound PCNA by the CRL4 ${ }^{\text {Cdt2 }}$ substrates ensures that protein degradation only occurs in S phase or after DNA damage, while the requirement of a specific residue at the +4 position of the PIP box in substrates of CRL4 ${ }^{\text {Cdt2 }}$ prevents other PCNA binding proteins from CRL4 ${ }^{\mathrm{Cdt} 2}$-mediated degradation (Havens and Walter, 2009). This may reflect a common mechanism adopted by CRL4 ${ }^{\text {Cdt2 }}$ ubiquitin ligase to specifically regulate the replication licensing system in a DNA synthesis-dependent manner.

Geminin, the Cdt1 inhibitor, is regulated by ubiquitylation, too (Fig. 2). It is a substrate of APC/C, another ubiquitin ligase that is a master regulator of the cell cycle (McGarry and Kirschner, 1998; Rape et al., 2006). The activity of geminin oscillates conversely with the activity of APC/C. High activity of $A P C / C$ in late $M$ phase and early $\mathrm{G} 1$ phase targets geminin for ubiquitylation-dependent proteolysis (McGarry and Kirschner, 1998), allowing activation of Cdt1 for subsequent replication licensing. Inactivation of the APC/C E3 ligase by the inhibitor, Emi1, in S and $\mathrm{G} 2$ phase upregulates the level of geminin. As a consequence, the licensing function of Cdt1 is inhibited and origin refiring is prohibited (Fig. 2) (Hsu et al.,
2002; Machida and Dutta, 2007). Therefore, in addition to the CRL ligase family, APC/C ubiquitin ligase is also a key regulator of DNA replication licensing during cell division. The activation of APC/C in S phase can induce replication refiring. Indeed, knocking down Emi1 through RNA interference (RNAi) triggers massive rereplication that can be attenuated by the inactivation of APC/C by co-silencing Cdh 1 and Cdc20 (Machida and Dutta, 2007). Although Geminin is degraded through ubiquitylation by APC/C in mitosis, interestingly, not all geminin is degraded in late $\mathrm{M}$ phase. Li and Blow (2004) showed that a portion of polyubiquitylated geminin is inactivated but is not subjected to proteolysis. However, the mechanism is not clear.

In summary, ubiquitylation-dependent protein degradation plays essential roles in regulating DNA licensing. It controls DNA replication licensing either by adjusting the level of pre$\mathrm{RC}$ components through direct ubiquitylation and degradation or by indirectly targeting upstream regulators according to the progression of the cell cycle.

\section{ACETYLATION MEDIATES DNA REPLICATION LICENSING}

Protein acetylation/deacetylation is a newly found mechanism involved in regulating the pre-RC components. A study published recently indicates that, in addition to phosphorylation and ubiquitylation, Cdc6 can also be acetylated by histone acetyltransferase (HAT) Gcn5 (Paolinelli et al., 2009). In this study, Paolinelli and colleagues showed that Gcn5 interacts with Cdc6 and acetylates Cdc6 on lysine 92, 105 and 109 in vivo. Interestingly, the acetylation on these three lysine residues is required for phosphorylation on Ser 106 of Cdc6, a prerequisite for the cytoplasmic transportation and degradation of Cdc6. Further investigation demonstrated that acetylation of $\mathrm{Cdc} 6$ reduces its chromatin binding affinity and promotes S-phase progression. However, rereplication was not observed in cells expressing Cdc6 mutants that are either unable to be phosphorylated or acetylated (Paolinelli et al., 2009). This may be due to the fact that higher eukaryotic cells utilize multiple mechanisms to prevent rereplication (Arias and Walter, 2007). It has been shown that overexpression of both Cdt1 and Cdc6 can induce rereplication (Yanow et al., 2001; Sugimoto et al., 2009). It would be interesting to investigate whether those Cdc6 mutants could trigger more profound rereplication phenotypes.

Another DNA replication licensing factor, Cdt1, is regulated by acetylation and deacetylation as well. HDAC11, the smallest member of the human histone deacetylase (HDAC) family, is shown to interact with Cdt1 directly (Glozak and Seto, 2009). The authors further showed that Cdt1 is acetylated and that acetylation may be catalyzed by lysine acetyltransferase 2B and 3B (KAT2B and KAT3B). Acetylation reduces Cdt1 ubiquitylation and protects Cdt1 from ubiquitylation and the subsequent proteolysis. Although the 
authors did not address whether acetylation of Cdt1 regulates replication licensing, one may speculate that hyperacetylation of Cdt1 may result in DNA rereplication. This remains to be tested in the future.

Furthermore, Cdt1 has been shown to interact with another histone acetyltransferase, Hbo1 (Miotto and Struhl, 2008). It has long been known that Hbo1 interacts with both Orc1 and Mcm2 (Lizuka and Stillman, 1999; Burke et al., 2001). There is also evidence suggesting that Hbo1 is required for the loading of $\mathrm{MCM} 2-7$ at the replication origins and positively regulates replication licensing (Lizuka et al., 2006). However, the mechanism remained unknown. Recently, in studying how Hbo1 regulates replication licensing, Miotto and Struhl (2008) found that Hbo1 is recruited to the origins through direct interaction with Cdt 1 in G1 phase (Fig. 1). Moreover, the interaction between Cdt1 and $\mathrm{Hbo} 1$ is independent of geminin. While Hbo1 does not regulate the stability of $\mathrm{Cdt} 1$, it enhances rereplication induced by Cdt1 overexpression. Therefore, Hbo1 is believed to function as a coactivator of Cdt1. In support of this, an earlier study indicated that the acetytransferase activity of Hbo1 is required for MCM2-7 loading in both human cells and Xenopus egg extracts and that the replication defects in Xenopus egg extract depleted of $\mathrm{Hbo} 1$ could be rescued by adding excess Cdt1 (Lizuka et al., 2006). However, in vitro experiment suggests that Cdt1 may not be acetylated by Hbo1 (Glozak and Seto, 2009). Instead, Miotto and Struhl (2010) further showed that Hbo1 acetylates histone $\mathrm{H} 4$ at the origins, and that this modification is required for MCM2-7 loading. Interestingly, although geminin does not interfere with the recruitment of $\mathrm{Hbo} 1$ by $\mathrm{Cdt} 1$, it inhibits $\mathrm{Hbo1}$-dependent histone $\mathrm{H} 4$ acetylation at the origins, which prevents MCM2-7 loading (Miotto and Struhl, 2010). Since hyperacetylated histone H4 correlates with an opened chromatin structure, the acetylation of histone $\mathrm{H} 4$ by $\mathrm{Hbo} 1$ may create a chromatin environment that favors the binding of MCM2-7 to the origin; alternatively, the acetylated histone $\mathrm{H} 4$ may create binding sites for some unidentified factors that is required for the loading of MCM2-7. These possibilities need to be tested in the future. Nonetheless, these data suggest that chromatin structure may play a direct role in regulation of replication licensing. It also suggests that geminin may inhibit the replication licensing through inhibition of histone $\mathrm{H} 4$ acetylation and the subsequent MCM2-7 loading.

\section{REPLICATION LICENSING AND CANCER CHEMOTHERAPY}

Proper control of replication licensing is essential for rereplication prevention and contributes to the maintenance of genome integrity. Therefore, it is not surprising that dysregulation of DNA replication licensing factors is associated with many human diseases, including cancers. Indeed, recent studies have shown that dysregulation of the pre-RC components as well as the upstream regulators is found in many types of cancer cells, such as breast, brain, prostate, oral, colorectal, ovarian, kidney, bladder, and hematological cancers (for reviews, see Gonzalez et al., 2005; Hook et al., 2007; Williams and Stoeber, 2007; and the included citations).

Multiple lines of evidence support the idea that overexpression of oncogenes can induce rereplication. Activation of the oncogene H-RasV12 in normal human cells induces DNA rereplication, which, in turn, triggers the DNA-damage checkpoint response and induces cellular senescence $(\mathrm{Di}$ Micco et al., 2006). The cellular senescence results from the activation of DNA damage response triggered by Rasinduced DNA rereplication and the DNA hyper-replication is most likely due to the upregulation of Cdc6 by H-RasV12. Overexpression of cyclin E in U2OS cells results in elevated origin firing, an indication of DNA rereplication (Bartkova et al., 2006). A nondegradable mutant allele of Cyclin D1, D1T286A, which is also refractory to nuclear export, inhibits the expression of Cullin4, thereby suppressing the ubiquitin ligase activity of CRL4 ${ }^{\mathrm{Cdt} 2}$. As a result, the level of Cdt1 is upregulated and DNA rereplication occurs (Aggarwal et al., 2007). Interestingly, Cyclin D1-induced rereplication is accelerated by inactivation of p53 (Aggarwal et al., 2007), while p53 can prevent rereplication triggered by over-expressing both Cdt1 and Cdc6 (Vaziri et al., 2003). However, it is unclear whether p53 plays any role in rereplication induced in Cdt2silenced cells. Recent studies have suggested that pre-RC components themselves have oncogenic properties. For example, injection of $\mathrm{NIH} 3 \mathrm{~T} 3$ fibroblasts that overexpress Cdt1 into mice leads to the formation of tumors (Arentson et al., 2002). Moreover, Cdt1 overexpression in T-cells lacking functional p53 results in lymphoblastic lymphoma (Seo et al., 2005). Cdc6 has also been shown to have oncogenic properties (reviewed in Borlado and Mendez, 2008).

Despite these observed oncogenic characteristics of DNA replication licensing factors, it is still not clear whether elevated levels of pre-RC components in cancer cells are results or causes of carcinogenesis. Nonetheless, DNA replication is becoming a potential target for cancer therapy. The rationale is that upregulation of DNA replication licensing usually leads to rereplication and genome instability, therefore renders cells more susceptible to drug treatments that target DNA damage response pathways. An interesting paper published recently suggests that geminin may be an effective target for cancer therapy (Zhu and Depamphilis, 2009). In this study, the authors found that depletion of geminin in various cancer cells induced DNA rereplication and apoptosis, while depletion of geminin in normal cells had no effect on cell proliferation. As mentioned earlier, replication licensing is controlled by multiple mechanisms in normal cells, so that 
depletion of geminin alone is not able to induce rereplication. In some cancer cells, however, one or more pathways may be impaired due to the dysregulation of pre-RC components, therefore, depletion of geminin can be lethal.

Another promising cancer therapeutic target is the Cdc7 protein kinase which regulates the replication licensing components. Cdc7 is known to phosphorylate the MCM members and promote replication initiation and entry into $S$ phase (Masai and Arai, 2002). It also promotes pre-RC assembly by phosphorylating $\mathrm{Mcm} 2$ during cell cycle re-entry from quiescence (Chuang et al., 2009). Depletion of Cdc7 prevents MCM loading and induces replication checkpoint response and cell cycle arrest in normal cells but not in cancer cells, leading the cancer cells to progress through a defective $S$ phase and eventually undergo apoptosis (Montagnoli et al., 2004). By taking advantage of this property of Cdc7, Montagnoli and colleagues (2008) found a small-molecule inhibitor of Cdc7, named PHA-767491 that preferentially induces apoptosis in cancer cell lines. This compound primarily targets $\mathrm{Cdc} 7$ and inhibits its kinase activity. Treating various cancer cell lines with PHA-767491 inhibits phosphorylation of MCM2-7 complex by Cdc7, prevents the initiation of DNA synthesis and results in apoptotic cell death, while treating primary fibroblasts with PHA-767491 only causes cell cycle arrest. In addition to PHA-767491, several other potent Cdc7 inhibitors have also been developed by a few pharmaceutical companies, and some of the inhibitors are currently under clinical trials (Swords et al., 2010).

In summary, the discovery that targeting the DNA replication licensing system results in selective killing of cancer cells is novel and exciting. These findings greatly encourage development of novel anticancer drugs and may establish a new mode for chemotherapy.

\section{VIRAL INFECTION DISRUPTS DNA REPLICATION LICENSING IN THE HOST CELLS}

Most viruses reproduce themselves by utilizing the DNA replication machinery of the host cells. Recently, more and more evidence suggests that, while busily replicating themselves using the host replication machinery, several types of viruses also interfere with the DNA replication of the host cells.

Early studies on Simian virus 40 (SV40) found that infection of SV40 is able to induce cellular DNA synthesis in monkey kidney cells (Hatanaka and Dulbecco, 1966) and mouse 3T3 cells (Gershon et al., 1966). It was later confirmed that SV40 infected cells escape cell cycle control and initiate multiple rounds of DNA replication within a single cycle, leading to endoreduplication of the host genome. The viral T antigen protein of SV40 is required for this endoreduplication phenotype (Friedrich et al., 1992, 1994; Perry and Lehman, 1998). Recently, a study found that $T$ antigen exerts its hyperreplication effect through targeting Nbs1, an essential protein that is involved in sensing DNA damage and suppressing inappropriate DNA rereplication in the cells (Wu et al., 2004). Another interesting study reported that, despite the fact that active rereplication occurs in SV40infected monkey kidney cells, low levels of $\mathrm{Mcm} 2$ and $\mathrm{Mcm} 3$ were found to associate with the chromatin after cells passing through $\mathrm{S}$ phase, the same in uninfected cells. This suggests that reloading of $\mathrm{MCM}$ proteins to the chromatin is not required for SV40-induced rereplication of the host DNA, further indicating that novel cellular replication licensing pathway(s) may be activated when cells are infected by SV40 (Friedrich et al., 2005).

Another example of virus infection regulating host DNA replication licensing comes from research on hepatitis $B$ virus (HBV). HBV is known to cause hepatitis and hepatocellular carcinoma (Beasley et al., 1981). The HBV X (HBX) protein, which is essential for viral replication (Zoulim et al., 1994), regulates gene expression as well as other cellular processes (Andrisani and Barnabas, 1999; Bouchard and Schneider, 2004). Recently, Rakotomalala and colleagues (2008) indentified a new function of HBX in regulating replication licensing in the host cells. They found that expression of HBX in an immortalized mouse hepatocyte cell line induced DNA rereplication. Further study demonstrated that expression of HBX resulted in elevated level of Cdt1 and Cdc6, while the level of geminin was downregulated. Perturbation of the balance between Cdt1 and geminin by HBX may be a direct cause of DNA rereplication in this case. Although the authors did not address how HBX deregulates the licensing factors, the significantly altered mRNA levels of Cdt1, Cdc6 and geminin suggest that HBX may exert its effect by regulating transcription of these genes (Rakotomalala et al., 2008). Alternatively, the fact that HBX is able to interact with Ddb1, a common linker protein in the CUL4 ubiquitin complex, and disrupt CRL4 ${ }^{\text {Cdt2 }}$ ubiquitin ligase-mediated proteolysis pathway (Bontron et al., 2002), suggests that HBX may induce rereplication by preventing degradation of Cdt1 and p21, which are both over-produced when CRL4 ${ }^{\mathrm{Cdt} 2}$ is inactivated (Jin et al., 2006; Kim et al., 2008).

Viruses may also interfere with their host cells by inhibiting cellular DNA replication. For example, human cytomegalovirus (HCMV) was shown to inhibit G1/S progression and cellular DNA synthesis (Bresnahan et al., 1996; Lu and Shenk, 1996; Dittmer and Mocarski, 1997). In HCMV infected human fibroblast cells, Cdt1 is downregulated and the expression of MCM proteins is delayed; whereas Cdc6 and geminin are both upregulated. The pre-RC assembly is blocked at the step of MCM loading (Biswas et al., 2003). These data suggest that HCMV suppresses cellular DNA synthesis by impairing replication licensing. Consistent with these results, HCMV infection also leads to inactivation of the $A P C^{C d h 1}$ E3 ligase, resulting in accumulation of Cdc6 and geminin (Tran et al., 2008). These data partially explain how HCMV infection inhibits DNA synthesis of the host cells. A 
similar inhibitory effect of host cell DNA replication was also detected with the human papillomavirus (HPV) E4 protein, which prevents MCM loading to the pre-RC complex by functioning together with Cdc6 through an unknown mechanism (Roberts et al., 2008).

Interference with host cell DNA replication by viruses is not limited to mammalian cells. A recent study found that the plant geminivirus rep protein can induce rereplication when introduced into fission yeast (Kittelmann et al., 2009). Since it is believed that disruption of host cell DNA replication may help divert the host cell machinery toward viral replication, understanding how virus infection disrupts host DNA replication licensing system may contribute to the development of new treatments for virus infection.

\section{CONCLUSION}

Proper regulation of DNA replication licensing is crucial to the fate of cells. Thus far, large efforts have been devoted to understand the mechanisms of how cells regulate replication licensing and prevent DNA rereplication within a single cell cycle. These mechanisms can be classified in three categories: inhibiting the activities of licensing proteins through posttranslational modifications or inhibitor binding, unloading the licensing proteins from chromatin, and downregulating the level of the licensing proteins when cells exit $S$ phase. Posttranslational modifications, including phosphorylation, ubiquitylation and acetylation, have been shown to play important roles in executing these mechanisms. In the future, it would be interesting to investigate whether other types of protein modification, such as methylation and SUMOylation, are involved in regulation of replication licensing. On the other hand, with the discovery of new factors (such as Mcm9, p21 and Hbo1) that are involved in licensing regulation, one can envision that additional pathways remain to be uncovered. Since dysregulation of the licensing process is tightly linked to tumorigenesis and virus infection, understanding cellular mechanisms that regulate DNA replication licensing will greatly benefit the chemotherapies of human diseases.

\section{ACKNOWLEDGEMENTS}

We thank Dr. Linda Guarino and Dr. Ju-Mei Li for critical reading of the manuscript. We apologize to those whose work has not been cited due to space limitation. Research in our laboratory is supported in part by The Welch Foundation. J.J. is a Pew Scholar.

\section{ABBREVIATIONS}

APC/C, anaphase-promoting complex/cyclosome; Cdc6, cell division cycle 6; CDK, cyclin dependent kinase; $\mathrm{CHO}$ cells, Chinese hamster ovary cells; CRL, Cullin-RING E3 superfamily; HAT, histone acetyltransferase; HDAC, histone deacetylase; Hbo1, histone acetyltransferase bound to Orc1; HBV, hepatitis B virus; HBX, HBV X; HCMV, human cytomegalovirus; HPV, human papillomavirus; KAT, lysine acetyltransferase; MCM, mini chromosome maintenance; ORC, origin recognition complex; PCNA ${ }^{\text {Chromatin }}$, chromatin-bound proliferating cell nuclear antigen; pre-RC, prereplication complex; RNAi, RNA interference; SV40, Simian virus 40

\section{REFERENCES}

Abbas, T., Sivaprasad, U., Terai, K., Amador, V., Pagano, M., and Dutta, A. (2008). PCNA-dependent regulation of p21 ubiquitylation and degradation via the $\mathrm{CRL} 4^{\mathrm{Cdt} 2}$ ubiquitin ligase complex. Genes Dev 22, 2496-2506.

Aggarwal, P., Lessie, M.D., Lin, D.I., Pontano, L., Gladden, A.B., Nuskey, B., Goradia, A., Wasik, M.A., Klein-Szanto, A.J., Rustgi, A. K., et al. (2007). Nuclear accumulation of cyclin D1 during $S$ phase inhibits Cul4-dependent Cdt1 proteolysis and triggers p53dependent DNA rereplication. Genes Dev 21, 2908-2922.

Amador, V., Ge, S., Santamaria, P.G., Guardavaccaro, D., and Pagano, M. (2007). APC/C(Cdc20) controls the ubiquitin-mediated degradation of p21 in prometaphase. Mol Cell 27, 462-473.

Andrisani, O.M., and Barnabas, S. (1999). The transcriptional function of the hepatitis $B$ virus $X$ protein and its role in hepatocarcinogenesis. Int J Oncol 15, 373-379.

Arentson, E., Faloon, P., Seo, J., Moon, E., Studts, J.M., Fremont, D. $\mathrm{H}$., and Choi, K. (2002). Oncogenic potential of the DNA replication licensing protein CDT1. Oncogene 21, 1150-1158.

Arias, E.E., and Walter, J.C. (2005). Replication-dependent destruction of Cdt1 limits DNA replication to a single round per cell cycle in Xenopus egg extracts. Genes Dev 19, 114-126.

Arias, E.E., and Walter, J.C. (2006). PCNA functions as a molecular platform to trigger Cdt1 destruction and prevent re-replication. Nat Cell Biol 8, 84-90.

Arias, E.E., and Walter, J.C. (2007). Strength in numbers: preventing rereplication via multiple mechanisms in eukaryotic cells. Genes Dev 21, 497-518.

Ballabeni, A., Melixetian, M., Zamponi, R., Masiero, L., Marinoni, F., and Helin, K. (2004). Human geminin promotes pre-RC formation and DNA replication by stabilizing CDT1 in mitosis. EMBO $\mathrm{J} 23$, 3122-3132.

Bartkova, J., Rezaei, N., Liontos, M., Karakaidos, P., Kletsas, D., Issaeva, N., Vassiliou, L.V., Kolettas, E., Niforou, K., Zoumpourlis, V.C., et al. (2006). Oncogene-induced senescence is part of the tumorigenesis barrier imposed by DNA damage checkpoints. Nature 444, 633-637.

Beasley, R.P., Hwang, L.Y., Lin, C.C., and Chien, C.S. (1981). Hepatocellular carcinoma and hepatitis $B$ virus. A prospective study of 22707 men in Taiwan. Lancet 2, 1129-1133.

Bell, S.P., and Dutta, A. (2002). DNA replication in eukaryotic cells. Annu Rev Biochem 71, 333-374.

Biswas, N., Sanchez, V., and Spector, D.H. (2003). Human cytomegalovirus infection leads to accumulation of geminin and inhibition of the licensing of cellular DNA replication. J Virol 77, 2369-2376.

Bontron, S., Lin-Marq, N., and Strubin, M. (2002). Hepatitis B virus X protein associated with UV-DDB1 induces cell death in the nucleus and is functionally antagonized by UV-DDB2. J Biol Chem 277 , 38847-38854.

Borlado, L.R., and Mendez, J. (2008). CDC6: from DNA replication to cell cycle checkpoints and oncogenesis. Carcinogenesis 29, 237-243. 
Bornstein, G., Bloom, J., Sitry-Shevah, D., Nakayama, K., Pagano, M., and Hershko, A. (2003). Role of the SCF ${ }^{\text {Skp2 }}$ ubiquitin ligase in the degradation of $\mathrm{p} 21^{\mathrm{Cip} 1}$ in S phase. J Biol Chem 278, 25752-25757.

Bouchard, M.J., and Schneider, R.J. (2004). The enigmatic X gene of hepatitis B virus. J Virol 78, 12725-12734.

Bresnahan, W.A., Boldogh, I., Thompson, E.A., and Albrecht, T. (1996). Human cytomegalovirus inhibits cellular DNA synthesis and arrests productively infected cells in late G1. Virology 224, 150-160.

Burke, T.W., Cook, J.G., Asano, M., and Nevins, J.R. (2001). Replication factors MCM2 and ORC1 interact with the histone acetyltransferase HBO1. J Biol Chem 276, 15397-15408.

Chen, X., Barton, L.F., Chi, Y., Clurman, B.E., and Roberts, J.M. (2007). Ubiquitin-independent degradation of cell-cycle inhibitors by the REGgamma proteasome. Mol Cell 26, 843-852.

Chuang, L.C., Teixeira, L.K., Wohlschlegel, J.A., Henze, M., Yates, J. R., Mendez, J., and Reed, S.I. (2009). Phosphorylation of Mcm2 by $\mathrm{Cdc} 7$ promotes pre-replication complex assembly during cell-cycle re-entry. Mol Cell 35, 206-216.

Delmolino, L.M., Saha, P., and Dutta, A. (2001). Multiple mechanisms regulate subcellular localization of human CDC6. J Biol Chem 276, 26947-26954.

DePamphilis, M.L. (2005). Cell cycle dependent regulation of the origin recognition complex. Cell Cycle 4, 70-79.

DePamphilis, M.L., Blow, J.J., Ghosh, S., Saha, T., Noguchi, K., and Vassilev, A. (2006). Regulating the licensing of DNA replication origins in metazoa. Curr Opin Cell Biol 18, 231-239.

Di Micco, R., Fumagalli, M., Cicalese, A., Piccinin, S., Gasparini, P., Luise, C., Schurra, C., Garre, M., Nuciforo, P.G., Bensimon, A., et al. (2006). Oncogene-induced senescence is a DNA damage response triggered by DNA hyper-replication. Nature 444 , 638-642.

Dittmer, D., and Mocarski, E.S. (1997). Human cytomegalovirus infection inhibits G1/S transition. J Virol 71, 1629-1634.

Drury, L.S., Perkins, G., and Diffley, J.F. (1997). The Cdc4/34/53 pathway targets Cdc6p for proteolysis in budding yeast. EMBO J 16, 5966-5976.

Elsasser, S., Chi, Y., Yang, P., and Campbell, J.L. (1999). Phosphorylation controls timing of Cdc6p destruction: A biochemical analysis. Mol Biol Cell 10, 3263-3277.

Friedrich, T.D., Bedner, E., Darzynkiewicz, Z., and Lehman, J.M. (2005). Distinct patterns of MCM protein binding in nuclei of $S$ phase and rereplicating SV40-infected monkey kidney cells. Cytometry A 68, 10-18.

Friedrich, T.D., Laffin, J., and Lehman, J.M. (1992). Simian virus 40 large T-antigen function is required for induction of tetraploid DNA content during lytic infection. J Virol 66, 4576-4579.

Friedrich, T.D., Laffin, J., and Lehman, J.M. (1994). Inductionof tetraploid DNA content by Simian virus 40 is dependent on Tantigen function in the $\mathrm{G} 2$ phase of the cell cycle. J Virol 68, 4028-4030.

Gershon, D., Sachs, L., and Winocour, E. (1966). The induction of cellular DNA synthesis by simian virus 40 in contactinhibited and in X-irradiated cells. Proc Natl Acad Sci U S A 56, 918-925.

Glozak, M.A., and Seto, E. (2009). Acetylation/deacetylation modulates the stability of DNA replication licensing factor Cdt1. J Biol
Chem 284, 11446-11453.

Gonzalez, M.A., Tachibana, K.E., Laskey, R.A., and Coleman, N. (2005). Control of DNA replication and its potential clinical exploitation. Nat Rev Cancer 5, 135-141.

Hatanaka, M., and Dulbecco, R. (1966). Induction of DNA synthesis by SV40. Proc Natl Acad Sci U S A 56, 736-740.

Havens, C.G., and Walter, J.C. (2009). Docking of a specialized PIP Box onto chromatin-bound PCNA creates a degron for the ubiquitin ligase CRL4 ${ }^{\text {Cdt2 }}$. Mol Cell 35, 93-104.

Hook, S.S., Lin, J.J., and Dutta, A. (2007). Mechanisms to control rereplication and implications for cancer. Curr Opin Cell Biol 19, 663-671.

Hsu, J.Y., Reimann, J.D., Sorensen, C.S., Lukas, J., and Jackson, P. K. (2002). E2F-dependent accumulation of hEmi1 regulates $S$ phase entry by inhibiting APC(Cdh1). Nat Cell Biol 4, 358-366.

$\mathrm{Hu}$, J., McCall, C.M., Ohta, T., and Xiong, Y. (2004). Targeted ubiquitination of CDT1 by the DDB1-CUL4A-ROC1 ligase in response to DNA damage. Nat Cell Biol 6, 1003-1009.

$\mathrm{Hu}, \mathrm{J}$., and Xiong, Y. (2006). An evolutionarily conserved function of proliferating cell nuclear antigen for Cdt1 degradation by the Cul4Ddb1 ubiquitin ligase in response to DNA damage. J Biol Chem 281, 3753-3756.

Jiang, W., Wells, N.J., and Hunter, T. (1999). Multistep regulation of DNA replication by Cdk phosphorylation of HsCdc6. Proc Natl Acad Sci U S A 96, 6193-6198.

Jin, J., Arias, E.E., Chen, J., Harper, J.W., and Walter, J.C. (2006). A family of diverse Cul4-Ddb1-interacting proteins includes Cdt2, which is required for $S$ phase destruction of the replication factor Cdt1. Mol Cell 23, 709-721.

Kim, J., Feng, H., and Kipreos, E.T. (2007). C. elegans CUL-4 prevents rereplication by promoting the nuclear export of CDC-6 via a CKI-1-dependent pathway. Curr Biol 17, 966-972.

Kim, Y., and Kipreos, E.T. (2007). The Caenorhabditis elegans replication licensing factor CDT-1 is targeted for degradation by the CUL-4/DDB-1 complex. Mol Cell Biol 27, 1394-1406.

Kim, J., and Kipreos, E.T. (2008). Control of the Cdc6 replication licensing factor in metazoa: the role of nuclear export and the CUL4 ubiquitin ligase. Cell Cycle 7, 146-150.

Kim, Y., Starostina, N.G., and Kipreos, E.T. (2008). The CRL4 $4^{\text {Cdt2 }}$ ubiquitin ligase targets the degradation of $\mathrm{p} 21^{\mathrm{Cip} 1}$ to control replication licensing. Genes Dev 22, 2507-2519.

Kittelmann, K., Rau, P., Gronenborn, B., and Jeske, H. (2009). Plant geminivirus rep protein induces rereplication in fission yeast. J Virol 83, 6769-6778.

Labib, K., Diffley, J.F., and Kearsey, S.E. (1999). G1-phase and Btype cyclins exclude the DNA-replication factor $\mathrm{Mcm} 4$ from the nucleus. Nat Cell Biol 1, 415-422.

Lewin, B. (2000). Genes VII. Oxford University Press: New York.

$\mathrm{Li}, \mathrm{A}$., and Blow, J.J. (2004). Non-proteolytic inactivation of geminin requires CDK-dependent ubiquitination. Nat Cell Biol 6, 260-267.

Li, C.J., Vassilev, A., and DePamphilis, M.L. (2004). Role for Cdk1 (Cdc2)/cyclin $A$ in preventing the mammalian origin recognition complex's largest subunit (Orc1) from binding to chromatin during mitosis. Mol Cell Biol 24, 5875-5886.

Li, X., Amazit, L., Long, W., Lonard, D.M., Monaco, J.J., and O'Malley, B.W. (2007). Ubiquitin- and ATP-independent proteolytic turnover of p21 by the REGgamma-proteasome pathway. Mol Cell 26, 831-842. 
Li, X., Zhao, Q., Liao, R., Sun, P., and Wu, X. (2003). The SCF(Skp2) ubiquitin ligase complex interacts with the human replication licensing factor Cdt1 and regulates Cdt1 degradation. J Biol Chem 278, 30854-30858.

Liku, M.E., Nguyen, V.Q., Rosales, A.W., Irie, K., and Li, J.J. (2005). CDK phosphorylation of a novel NLS-NES module distributed between two subunits of the MCM2-7 complex prevents chromosomal rereplication. Mol Biol Cell 16, 5026-5039.

Liu, E., Li, X., Yan, F., Zhao, Q., and Wu, X. (2004). Cyclin-dependent kinases phosphorylate human Cdt1 and induce its degradation. J Biol Chem 279, 17283-17288.

Lizuka, M., Matsui, T., Takisawa, H., and Smith, M.M. (2006). Regulation of replication licensing by acetyltransferase Hbo1. Mol Cell Biol 26, 1098-1108.

lizuka, M., and Stillman, B. (1999). Histone acetyltransferase HBO1 interacts with the ORC1 subunit of the human initiator protein. $J$ Biol Chem 274, 23027-23034.

Lovejoy, C.A., Lock, K., Yenamandra, A., and Cortez, D. (2006). DDB1 maintains genome integrity through regulation of Cdt1. Mol Cell Biol 26, 7977-7990.

Lu, M., and Shenk, T. (1996). Human cytomegalovirus infection inhibits cell cycle progression at multiple points, including the transition from G1 to S. J Virol 70, 8850-8857.

Lutzmann, M., and Mechali, M. (2008). MCM9 binds Cdt1 and is required for the assembly of prereplication complexes. Mol Cell 31, 190-200.

Machida, Y.J., and Dutta, A. (2007). The APC/C inhibitor, Emi1, is essential for prevention of rereplication. Genes Dev 21, 184-194.

Masai, H., and Arai, K. (2002). Cdc7 kinase complex: a key regulator in the initiation of DNA replication. J Cell Physiol 190, 287-296.

McGarry, T.J., and Kirschner, M.W. (1998). Geminin, an inhibitor of DNA replication, is degraded during mitosis. Cell 93, 1043-1053.

Mendez, J., Zou-Yang, X.H., Kim, S.Y., Hidaka, M., Tansey, W.P., and Stillman, B. (2002). Human origin recognition complex large subunit is degraded by ubiquitin-mediated proteolysis after initiation of DNA replication. Mol Cell 9, 481-491.

Mimura, S., Seki, T., Tanaka, S., and Diffley, J.F. (2004). Phosphorylation-dependent binding of mitotic cyclins to Cdc6 contributes to DNA replication control. Nature 431, 1118-1123.

Miotto, B., and Struhl, K. (2008). HBO1 histone acetylase is a coactivator of the replication licensing factor Cdt1. Genes Dev 22, 2633-2638.

Miotto, B., and Struhl, K. (2010). HBO1 histone acetylase activity is essential for DNA replication licensing and inhibited by Geminin. Mol Cell 37, 57-66.

Moldovan, G.L., Pfander, B., and Jentsch, S. (2007). PCNA, the maestro of the replication fork. Cell 129, 665-679.

Montagnoli, A., Tenca, P., Sola, F., Carpani, D., Brotherton, D., Albanese, C., and Santocanale, C. (2004). Cdc7 inhibition reveals a p53-dependent replication checkpoint that is defective in cancer cells. Cancer Res 64, 7110-7116.

Montagnoli, A., Valsasina, B., Croci, V., Menichincheri, M., Rainoldi, S., Marchesi, V., Tibolla, M., Tenca, P., Brotherton, D., Albanese, C., et al. (2008). A Cdc7 kinase inhibitor restricts initiation of DNA replication and has antitumor activity. Nat Chem Biol 4, 357-365.

Nguyen, V.Q., Co, C., Irie, K., and Li, J.J. (2000). Clb/Cdc28 kinases promote nuclear export of the replication initiator proteins MCM2-7. Curr Biol 10, 195-205.
Nguyen, V.Q., Co, C., and Li, J.J. (2001). Cyclin-dependent kinases prevent DNA re-replication through multiple mechanisms. Nature 411, 1068-1073.

Nishitani, H., and Lygerou, Z. (2002). Control of DNA replication licensing in a cell cycle. Genes Cells 7, 523-534.

Nishitani, H., Shiomi, Y., lida, H., Michishita, M., Takami, T., and Tsurimoto, T. (2008). CDK inhibitor p21 is degraded by a proliferating cell nuclear antigen-coupled Cul4-DDB $1^{\text {Cdt2 }}$ pathway during $S$ phase and after UV irradiation. J Biol Chem 283, 29045-29052.

Nishitani, H., Sugimoto, N., Roukos, V., Nakanishi, Y., Saijo, M., Obuse, C., Tsurimoto, T., Nakayama, K.I., Nakayama, K., Fujita, M., et al. (2006). Two E3 ubiquitin ligases, SCF-Skp2 and DDB1Cul4, target human Cdt1 for proteolysis. EMBO J 25, 1126-1136.

Paolinelli, R., Mendoza-Maldonado, R., Cereseto, A., and Giacca, M. (2009). Acetylation by GCN5 regulates CDC6 phosphorylation in the $S$ phase of the cell cycle. Nat Struct Mol Biol 16, $412-420$.

Perry, M.B., and Lehman, J.M. (1998). Activities of SV40 T antigen necessary for the induction of tetraploid DNA content in permissive CV-1 cells. Cytometry 31, 251-259.

Petersen, B.O., Lukas, J., Sorensen, C.S., Bartek, J., and Helin, K. (1999). Phosphorylation of mammalian CDC6 by Cyclin A/CDK2 regulates its subcellular localization. EMBO J 18, 396-410.

Pickart, C.M. (2004). Back to the future with ubiquitin. Cell 116, 181-190.

Prives, C., and Gottifredi, V. (2008). The p21 and PCNA partnership: a new twist for an old plot. Cell Cycle 7, 3840-3846.

Rakotomalala, L., Studach, L., Wang, W.H., Gregori, G., Hullinger, R. L., and Andrisani, O. (2008). Hepatitis B virus $X$ protein increases the Cdt1-to-geminin ratio inducing DNA re-replication and polyploidy. J Biol Chem 283, 28729-28740.

Ralph, E., Boye, E., and Kearsey, S.E. (2006). DNA damage induces Cdt1 proteolysis in fission yeast through a pathway dependent on Cdt2 and Ddb1. EMBO Rep 7, 1134-1139.

Rape, M., Reddy, S.K., and Kirschner, M.W. (2006). The processivity of multiubiquitination by the APC determines the order of substrate degradation. Cell 124, 89-103.

Roberts, S., Kingsbury, S.R., Stoeber, K., Knight, G.L., Gallimore, P. H., and Williams, G.H. (2008). Identification of an arginine-rich motif in human papillomavirus type 1 E1;E4 protein necessary for E4-mediated inhibition of cellular DNA synthesis in vitro and in cells. J Virol 82, 9056-9064.

Saha, T., Ghosh, S., Vassilev, A., and DePamphilis, M.L. (2006). Ubiquitylation, phosphorylation and Orc2 modulate the subcellular location of Orc1 and prevent it from inducing apoptosis. J Cell Sci 119, 1371-1382.

Sansam, C.L., Shepard, J.L., Lai, K., lanari, A., Danielian, P.S., Amsterdam, A., Hopkins, N., and Lees, J.A. (2006). DTL/CDT2 is essential for both CDT1 regulation and the early G2/M checkpoint. Genes Dev 20, 3117-3129.

Senga, T., Sivaprasad, U., Zhu, W., Park, J.H., Arias, E.E., Walter, J. C., and Dutta, A. (2006). PCNA is a cofactor for Cdt1 degradation by CUL4/DDB1-mediated N-terminal ubiquitination. J Biol Chem 281, 6246-6252.

Seo, J., Chung, Y.S., Sharma, G.G., Moon, E., Burack, W.R., Pandita, T.K., and Choi, K. (2005). Cdt1 transgenic mice develop lymphoblastic lymphoma in the absence of p53. Oncogene 24, 
8176-8186.

Sugimoto, N., Tatsumi, Y., Tsurumi, T., Matsukage, A., Kiyono, T., Nishitani, H., and Fujita, M. (2004). Cdt1 phosphorylation by cyclin A-dependent kinases negatively regulates its function without affecting geminin binding. J Biol Chem 279, 19691-19697.

Sugimoto, N., Yoshida, K., Tatsumi, Y., Yugawa, T., Narisawa-Saito, M., Waga, S., Kiyono, T., and Fujita, M. (2009). Redundant and differential regulation of multiple licensing factors ensures prevention of re-replication in normal human cells. J Cell Sci 122, 1184-1191.

Swords, R., Mahalingam, D., O'Dwyer, M., Santocanale, C., Kelly, K., Carew, J., and Giles, F. (2010). Cdc7 kinase- A new target for drug development. Eur J Cancer 46, 33-40.

Tada, S., Li, A., Maiorano, D., Mechali, M., and Blow, J.J. (2001). Repression of origin assembly in metaphase depends on inhibition of RLF-B/Cdt1 by geminin. Nat Cell Biol 3, 107-113.

Takeda, D.Y., Parvin, J.D., and Dutta, A. (2005). Degradation of Cdt1 during $S$ phase is Skp2-independent and is required for efficient progression of mammalian cells through $\mathrm{S}$ phase. J Biol Chem 280, 23416-23423.

Tran, K., Mahr, J.A., Choi, J., Teodoro, J.G., Green, M.R., and Spector,. (2008). Accumulation of substrates of the anaphasepromoting complex (APC) during human cytomegalovirus infection is associated with the phosphorylation of Cdh1 and the dissociation and relocalization of APC subunits. J Virol 82, 529-537.

Vaziri, C., Saxena, S., Jeon, Y., Lee, C., Murata, K., Machida, Y.,
Wagle, N., Hwang, D.S., and Dutta, A. (2003). A p53-dependent checkpoint pathway prevents rereplication. Mol Cell 11, 997-1008.

Williams, G.H., and Stoeber, K. (2007). Cell cycle markers in clinical oncology. Curr Opin Cell Biol 19, 672-679.

Wohlschlegel, J.A., Dwyer, B.T., Dhar, S.K., Cvetic, C., Walter, J.C., and Dutta, A. (2000). Inhibition of eukaryotic DNA replication by geminin binding to Cdt1. Science 290, 2309-2312.

Wu, X., Avni, D., Chiba, T., Yan, F., Zhao, Q., Lin, Y., Heng, H., and Livingston, D. (2004). SV40 Tantigen interacts with Nbs1 to disrupt DNA replication control. Genes Dev 18, 1305-1316.

Yanow, S.K., Lygerou, Z., and Nurse, P. (2001). Expression of Cdc18/ Cdc6 and Cdt1 during $\mathrm{G} 2$ phase induces initiation of DNA replication. EMBO J 20, 4648-4656.

Yu, Z.K., Gervais, J.L., and Zhang, H. (1998). Human CUL-1 associates with the SKP1/SKP2 complex and regulates p21 (CIP1/WAF1) and cyclin D proteins. Proc Natl Acad Sci U S A 95, 11324-11329.

Zhong, W., Feng, H., Santiago, F.E., and Kipreos, E.T. (2003). CUL-4 ubiquitin ligase maintains genome stability by restraining DNAreplication licensing. Nature 423, 885-889.

Zhu, W., and Depamphilis, M.L. (2009). Selective killing of cancer cells by suppression of geminin activity. Cancer Res 69, $4870-4877$.

Zoulim, F., Saputelli, J., and Seeger, C. (1994). Woodchuck hepatitis virus $X$ protein is required for viral infection in vivo. J Virol 68, 2026-2030. 